Tetrahedron Letters Nu.50, Pp. 5209-5213, 1968. Pergnmon Pres8. Printed in Graat Britain.

\author{
ALTE UND NEUE CYCLOADDITIONEN DER NITRILOXIDE \\ Manfred Christl und Rolf Huisgen \\ Institut fur Organische Chemfe der Universität Mlinchen
}

(Received in Jermany 6 September 1968; received in TK for publication 16 September 1968)

A. Acrylsäureester und Derivate

In schönen Arbetten haben Quilico und seine Schule (1) - Im wesent1ichen von 1945 - 1960 - die Cycloadditionen der Nitriloxide an CC-Doppe1- und CC-Dreifachbindungen erschlossen. Von wonigen Ausnahmen $(2,3)$ abgesehen, wurden die Anlagerungen des Benzonitriloxids an $\alpha, \beta$-ungesbttigte Carbonester als richtungsmälig eindeutig beschrieben. In dieser Eindeutigkeit sah Firestone (4) ein wichtiges Argument fur die Annahne diradikalischer Zwischenstufen bei 1.3Dipolaren Cycloaduitionen (5).

Als Vorarbeiten für die Ermittlung partieller Additionskonstanten wiederholten und ergänzten wir fruhere Versuche. Um die oligomerisation des Formonitriloxids (Knalisăure) und die Furoxan-Bildung aus höheren Nitriloxiden zurlickzudrängen, bedienten wir uns niedriger stationärkonzentration; diege wurde durch langsame Freisetzung des Nitriloxids aus den Hydroximsäure-Halogeniden mit Triathylamin in Gegenwart Uberschüssigen Dipolarophils erzielt (6). Ledig1ich 2.4.6Trimethyl- und 2.4.6-Trimethoxy-benzonitriloxid (7) sind stabil und lieferten hohe Adduktausbeuten. Die $\Delta^{2}$-Isoxazolin-carbonester wurden nach Hochvak.-Destillation der quantitativen NMR-Analyse unterworfen; anschliebend wurden metst die Stellungsisomeren dünschichtchramatographisch getrennt und anhand der NMR-Spektren aufgrund etnes grbßeren Erfahrungemeterials (8) zugeordnet.

Die Tab. I lehrt, daB schon Acrylsaure-methylester die Nitriloxide in belden Additionsrichtungen aufnimat; lediglich bel knallsăure und Pivalonitriloxid lag die zweite Additionsrichtung unter It. Auch Crotonabure-nethylester ist nicht inert gegenuber Benzonitriloxid (9), sondern 110 ferte $84 \%$ cycloaddukt. Seine hinter Acrylsäureester zuruckstehende dipolarophile Aktivitat hat zur Fol- 
<smiles>[R]C=CC(=O)OC</smiles>

TABELLE 1

Verhkitnisoe von $\Delta^{2}-I s o x a z 011 n-c a r b o n s k u r e-(5)$ - und -carbonskure-(4)-methylosternbei der Cycloaddition von Nitriloxiden an $\alpha, \beta$-ungesittigte Carbonester in Äther (fa11s Lชsungsmittel verwendet); $\not$ Gesamtausbeute in Klammern

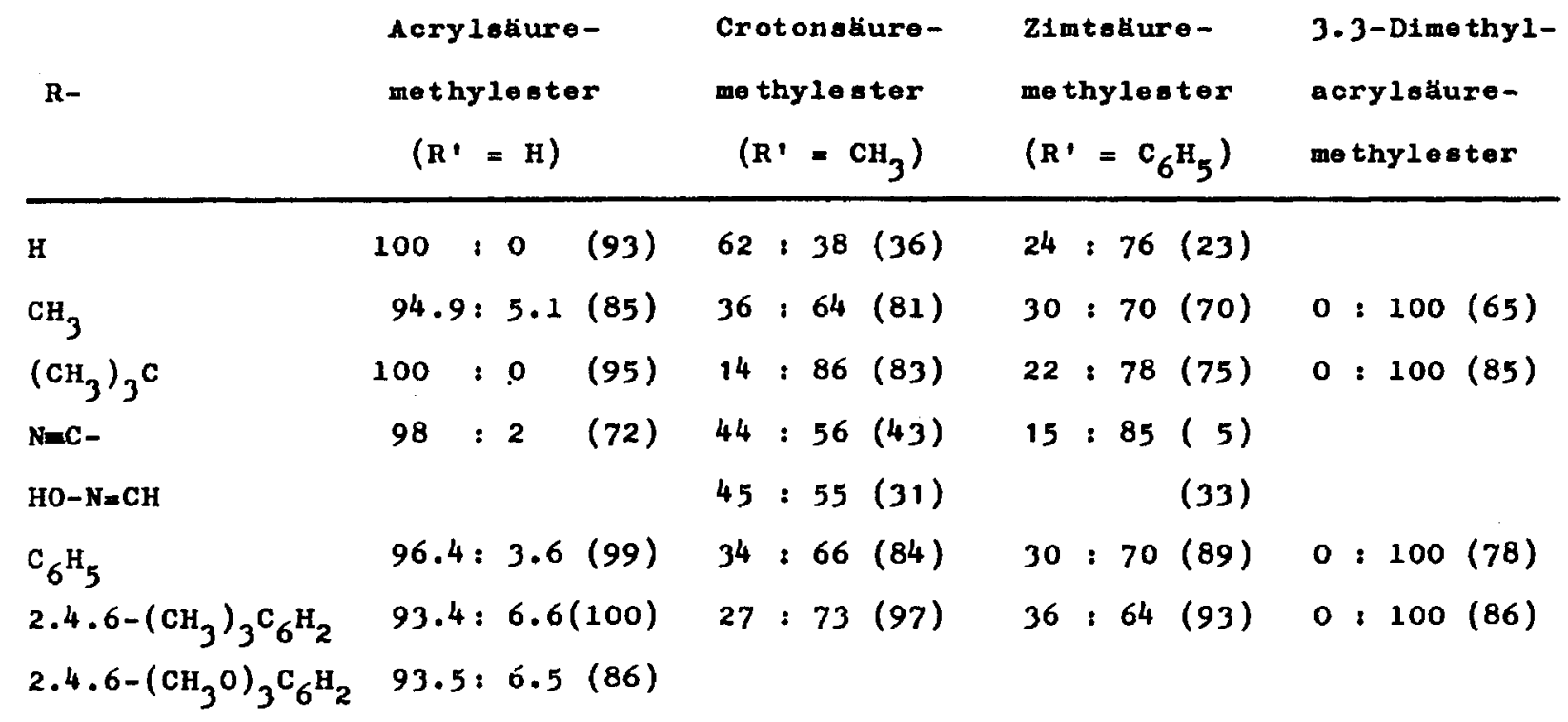

ge, daß Kna11saure teilwelse zum Isonitroso-acetonitriloxid dimerisiert und sich als solches an den Crotonsburesster anlagert (10). Bel diesem Dipolarophil stbBt man auf die größte Varlation des Verhaltnisses der Additionsrichtungen; energetisch bewegt sie sich allerdings auch nur innerhalb $\Delta \Delta G^{\ddagger}=1.3 \mathrm{kcal} / \mathrm{Mol}$. Mit schrittwelser Einfuhrung von Methylgruppen in den Acrylstureester trat der Vorzug der Bildung des Isoxazolin-5-carbonsaureesters zuruck; 3.3-Dimethyl-acrylsăure-methyleater erbrachte nur noch den 4-Carbonester. Man ist gene1gt, diese Umkehr in der orientierung dem Gegeneinander von sterischen und elektronischen Effekten zuzuschreiben. Man begegnet Schwlerigketten, da die Abstufung in den vertikalen Kolonnen der Tab. 1 keine einfache Bezlehung zum Raumbedarf von $R$ erkennen 1kBt. Man gewinnt vielmehr den Elndruck, dab das Orientierungeverhal- 
ten elne dem Nitriloxid-System immanente, von der Natur des $R$ nur untergeordnet beeinfluBte Eigenschaft ist.

B. Acetylen-carbonester

Aus den Cycloaditionen an acetylenieche Carbonester gingen aromatische Isoxazole in hohen Ausbeuten hervor (Tab. 2). Der Vorzug des 5-Carbonesters in den aus Propiolsäure-methylester entstehenden Isomerengemischen ist geringer a 1s bei der Anlagerung an Acryloaureester. Betm tbergang zu Tetrolsare- und Phenylpropiolsăureester erhielt man fast nur noch die Isoxazo1-4-carbonsăureester.<smiles>[R]C#CC(=O)OCc1onc([R])c1[R]</smiles>

\section{TABELLE 2}

Verhaltnisse von Isoxazol-carbonskure-(5)- und -carbonsaure-(4)-methylestern bei der Cycloaddition von Nitriloxiden an Acetylen-carbonester in Äther; $\%$ Gesamtausbeute in Klammern

\begin{tabular}{ccc} 
Propiolsaure- & Tetrolsure- & Phenylpropiol- \\
methylester & methylester & oburesster \\
$\left(R^{\prime}=\mathrm{H}\right)$ & $\left(\mathrm{R}^{\prime}=\mathrm{CH}_{3}\right)$ & $\left(\mathrm{R}^{\prime}=\mathrm{C}_{6} \mathrm{H}_{5}\right)$ \\
\hline
\end{tabular}

H

$84: 16(50)$

$\left(\mathrm{CH}_{3}\right)_{3} \mathrm{C}$

$91: 9(95)$

$\mathrm{N}=\mathrm{C}-$

$68: 32(69)$

$\mathrm{C}_{6} \mathrm{H}_{5}$

$72: 28$ (98)

$1.3: 98.7(83)$

$1.2: 98.8(93)$

$2.4 .6-\left(\mathrm{CH}_{3}\right)_{3} \mathrm{C}_{6} \mathrm{H}_{2}$

$28: 72(99)$

c. Cyclooctatetraen

Entgegen der fruher vermuteten Reaktionstrugheit gegentiber Benzonitriloxid (9) gelangten wir zu $83 \%$ des kristalilnen Cycloaddukts II, dessen tri- 
cyclische struktur aus dem NMR-Spektrum $\left(\operatorname{CDC} 1_{3}\right)$ abzulesen war. Ein S1ngulett (breit) fur 4 vinylprotonen erscheint be1 4.20 , die Multipletts fur 3a-H<smiles>CCCCCCCC1(OC)C2/C=C\C=C/C=C\C2ON1C</smiles>

I<smiles>C1=CC2C=CC2C=C1</smiles>

IV

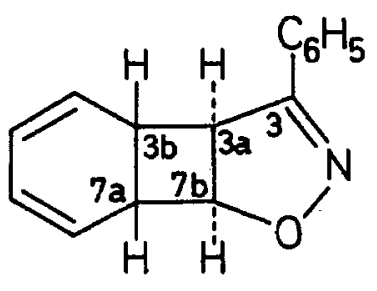

II

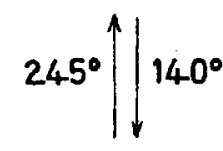<smiles>CCCCCc1ccon1</smiles>

III

gegen 5.72, fur $3 b-H$ und $7 a-H$ bei 6.66 und fír $7 b-H$ bei 4.90. Das gleiche Addukt II wurde jungst auch von Bianch1, Gandolf1 und Grinanger (11) beschrieben, die einen eleganten chemischen Strukturbeweis erbrachten. Bei $140^{\circ} \mathrm{m}$ Hochvak. trat neben die II-Thermolyse zu 3-Pheny1-18oxazol (III, 71\%) und Benzol elne Dimerisation zu 29\%; In Analogie zur Cyclohexadien-Dimerisation erscheint $v$ plausibel. Oberhalb des Schmelzpunktes $\left(243^{\circ}\right)$ spaltete auch V In III + Benzol. Die Italien. Autoren (11) lassen offen, ob Cyelooctatetraen oder Bicyclooctatrien (IV) alo Roaktionspartner des Benzonitriloxids dient. In uneerem Arbeitskreis wurde fruher gezeigt, dab das Valenztautomerie-Gleichgewicht des Cyclooctatetraens mit $0.01 \%$ IV orst oberhalb $90^{\circ}$ ausreichend mob11 1st (12). Als vir die Umsotzung dos Cyclooctatetraens mit Benzonitriloxid und die Aufarbeltung bel $\leqslant 10^{\circ}$ vornahmen, traten $1 \mathrm{~m}$ NMR-Spektrum neben den Signalen von II diejenigen des Cyclooctatrien-Abkömmlinge I auf. Nach 1-stindigon Erwärmen auf $80^{\circ}$ hat te sich I Innerhalb der NMR-Nachwe1sgrenze vollständig zu II 1somerisiert; die Ringabschnurung volizieht sich also erst auf der Cyclooctatrien-Stufe. tber ein II enteprechendes Knallsture-Addukt des Cyclooctatetraens berichteten wir 
In anderem Zusammenhang (10).

Der Deutschen Forschungsgemeinschaft und dem Fonds der Chemiachen Induotrie schulden wir fur die Förderung des Arbeiteprogramms Dank.

\section{LITERATUR}

1. tbersicht: A. Quilico In "Five- and Six-Membered Compounds with Nitrogen and Oxygen", S. 19, 99 (1962), The Chemistry of Heterocyolic Compounds, herausgegeben von A. Weissberger, Interscience Publiahers.

2. o- und p-Nitro-zimtester: F. Monforte und G. Lo Vecohio, Gazz.ohim.1tal. 83.416 (1953).

3. cis-Zimtester: P. Grunanger, C. Gandini und A. Quilico, Rend, Ist.Lomberd. sci.lett. 23, 467 (1959).

4. R.A. Firestone, J.Org.Chem 33, 2285 (1968).

5. Widerlegung: R. Huisgen, J.Org.Chem. 33. 2291 (1968).

6. R. Huiggen und W. Mack, Tetrahedron Letters 1961, 583.

7. C. Grundmeng und J.M. Dean, J.Org.Chem. 30, 2809 (1965).

8. R. Sustmann, R. Hulagen und H. Huber, Chom.Ber, 100, 1802 (1967).

9. Loe. cit. (1), S. $100 / 101$.

10. R. Hutegen und M. Christ1, Anger.Chem, 79, 471 (1967).

11. G. Bianch1, R. Gandolfi und P. Grinanger, Chimica é Induatria 49, 757 (1967).

12. R. Hulsgen, F. Mietzsch, G. Boche und H. Seldl in Orgenic Renction Mechan1sma, Spec.Pub1.Chem.Soc. [London] 19, 3 (1965). 